\title{
Duke's Discovery Genomics center rides pharmacogenomics wave
}

Last year, a research project led by David Goldstein of Duke University found that variations in the $I L 28 B$ gene had profound effects on how people with hepatitis C respond to treatment. More specifically, those with certain mutations were twice as likely to respond to prolonged drug therapy (Nature 461, 399-401, 2009). The link's impact on patient care and drug development served as a wake-up call for investigators to ramp up efforts to identify genetic variants associated with disease occurrence and treatment response.

Spurred by this homegrown insight, investigators at the Duke University School of Medicine in Durham, North Carolina decided to form Discovery Genomics. The new center-a collaboration between the Duke Clinical Research Institute (DCRI) and the university's Center for Human Genome Variation (CHGV)-launched in October. Although projects within the university are its main focus, Discovery Genomics also operates as a fee-for-service business for pharmaceutical companies interested in finding genetic variations associated with drug efficacy and toxicity.

The commercial packages include genomewide association studies and whole-exome sequencing to search for common and rare genetic variants, respectively, among DNA samples collected in clinical trials. Discovery

\section{Minipig, minipig, let me in}

Animal experimentation remains an essential component of drug safety testing. But that doesn't mean that pharmaceutical companies have to rely on the same old animal models. According to a panel of European experts, diminutive strains of domestic hogs called minipigs could provide more suitable platforms for toxicity testing than monkeys, dogs, rats and other species routinely used to gauge drug safety.

"There are many features of the minipig that make it more amenable for being a toxicology model," says Roy Forster, chief scientific officer of the French contract research organization CIT. "If you had to start the field of toxicology all over again and you didn't have the history and the tradition that we do, then the minipig would certainly be an animal that we would readily turn to."

Reporting in a series of eight papers published in the current issue of the Journal of Pharmacological and Toxicological Methods, Forster and his colleagues from the EU-funded RETHINK project argue that minipigs, which are markedly smaller than their farmyard porcine cousins, should become the lab animal of choice for testing new drugs. The minipigs are easier to house and carry fewer zoonotic diseases than nonhuman primates, they share more physiological and metabolic similarities to humans than other large mammals, their large litter sizes and quick development time make them easier to breed and they have more genetic and genomic background data available than for other animal models of toxicology. Although switching to minipigs would still involve animal experimentation, Forster argues that the data gleaned from each minipig will be more informative and predictive of human biology than existing models.

"Pigs are basically taking over the world as the large-animal model of choice," says Michael Swindle, a swine researcher at the Medical University of South Carolina in Charleston.

Elie Dolgin
Genomics will interpret the data and prepare publications authored by faculty, with industry ties disclosed.

The center's pharmacogenomic studies for drug efficacy cost about $\$ 500$ per sample. Those for toxicity, meanwhile, cost about $\$ 6,000$ per sample for wholeexome sequencing and $\$ 15,000$ per sample for genome-wide scans. According to Tom Kaminski, DCRI's manager of business strategy, the costs are competitive with wholly commercial entities offering similar services, such as Expression Analysis, also in Durham.

Discovery Genomics highlights its search for rare genetic variants as a pioneering approach. "Little by little, more people are [agreeing] that it's not common but rare variation that will be associated with many complex diseases," says Kevin Shianna, director of CHGV's genotyping facility.

But according to David Altshuler, a geneticist at Massachusetts General Hospital (MGH) in Boston, the approach echoes the technology-driven direction of pharmacogenomics overall. "It's not so much a change in strategy as an evolution of technology," says Altshuler. "Five years ago, there was no way to do the sequencing to study rare genetic variants."

Several other academic institutions have created engines to facilitate the incorporation of pharmacogenomics into clinical research. Similar efforts include MGH's Center for Human Genetic Research and the Penn Genome Frontiers Institute at the University of Pennsylvania in Philadelphia.

The Duke center is among the first to convert its faculty expertise and technological know-how into a business model. According to Kaminski, Discovery Genomics' strength lies with its storehouse of 200 full-coverage sequences to which new sequences can be compared, enabling researchers to glean meaningful data from a small number of new samples.

Still, some skepticism remains about the current approach. Christopher NewtonCheh, a cardiologist and geneticist at $\mathrm{MGH}$, cautions about the high potential for oversimplified or incorrect interpretations of the data. "Sometimes a result of a genetic variant in one tissue could counteract the result in another tissue," says Newton-Cheh. "[Pharmacogenomics] may open potential avenues... but it also has the potential to close [them]." 